\title{
Backward Linear Control Systems on Time Scales*
}

\author{
Ewa Pawłuszewicz ${ }^{a, b}$ \\ ewa@ua.pt, epaw@pb.edu.pl \\ Delfim F. M. Torres ${ }^{a}$ \\ delfim@ua.pt \\ ${ }^{a}$ Department of Mathematics, University of Aveiro \\ 3810-193 Aveiro, Portugal \\ ${ }^{b}$ Faculty of Computer Science, Białystok University of Technology \\ 15-351 Białystok, Poland
}

\begin{abstract}
We show how a linear control systems theory for the backward nabla differential operator on an arbitrary time scale can be obtained via Caputo's duality. More precisely, we consider linear control systems with outputs defined with respect to the backward jump operator. Kalman criteria of controllability and observability, as well as realizability conditions, are proved.
\end{abstract}

Keywords: time scales; duality; linear time varying control systems; controllability; observability; realizability.

Mathematics Subject Classification 2010: 34N05, 93B05, 93B07, 93B15.

\section{Introduction}

The theory of linear control systems of both continuous- and discrete-time cases is a subject well developed - see, e.g., (Kalman et al., 1969; Wolovich and Elliott, 1983; Olsder and van der Woude, 2005; Zabczyk, 2008) and references therein. It can be noticed that many results obtained in both discrete and continuous cases are similar or even identical. Recently, many problems in control theory have been generalized to time scales (DaCunha, 2004, 2005; Bartosiewicz and Pawłuszewicz, 2006; Bartosiewicz et al., 2006, 2007; Jackson, 2007; Davis et al., 2009; Pawłuszewicz and Torres, 2010). The mathematics of time scales was born in 1988 (Aulbach and Hilger, 1990), providing a rich calculus that unifies and extends the theories of difference and differential equations (Bohner and Peterson, 2001). A time scale is a model of time. Besides the standard cases of the whole real line (continuous-time case) and all integers (discrete-time case) there are many other models of time included, e.g., the time scale $\mathbb{P}_{a, b}=\bigcup_{k=0}^{\infty}[k(a+b), k(a+b)+a], q$-scales, quantum time scales (objects with nonuniform domains), and many others - see (Bohner and Peterson, 2001, 2003). However, the discrete-time systems on time scales are based on the difference operator and not on the more conventional shift operator. Note that the difference operator description provides a smooth transition from sampled-data algorithms to their continuous-time counterparts (Goodwin et al., 2001). In order to deal with non-traditional applications in areas such as medicine, economics, or engineering, where the system dynamics are described on a time scale partly continuous and partly discrete, or to accommodate non-uniform sampled systems, one needs to work with systems defined on a time scale - see, e.g., (Atici et al., 2006; Atici and Uysal, 2008).

The study of control systems defined on an arbitrary time scale is a six years old emerging research area under strong current research (DaCunha, 2004, 2005; Bartosiewicz and Pawłuszewicz,

*Submitted November 11, 2009; Revised March 28, 2010; Accepted April 03, 2010; for publication in the International Journal of Control. 
2006; Bartosiewicz et al., 2006, 2007; Jackson, 2007; Ferreira and Torres, 2008; Jackson, 2008; Bartosiewicz and Pawluszewicz, 2008; Davis et al., 2009; Kotta et al., 2009; Pawłuszewicz and Torres, 2010), motivated by multidisciplinary applications that require simultaneous modeling of discrete and continuous data (Seiffertt et al., 2008). In (Bartosiewicz and Pawłuszewicz, 2006) the question of realizability of linear time-invariant control systems defined on time scales is studied. Main result shows how to construct a state space representation of an abstract input/output map and gives conditions for this map to allow such a representation. It is also proved that classical Kalman conditions (Kalman et al., 1969) are still valid for systems on time scales. The assumption of regressivity for the considered control systems is dropped. This assumption implies existence and uniqueness of both forward and backward solutions of linear delta differential equations (Bohner and Peterson, 2001). In problems that are studied in (Bartosiewicz and Pawtuszewicz, 2006) only forward solutions are needed and they exist without the regressivity hypothesis. In (Davis et al., 2009) it is developed, under the regressivity assumption, the foundational notions of controllability, observability, and realizability of time-varying linear control systems defined on an arbitrary time scale. The proposed generalized framework has already shown promising applications (Davis et al., 2009). A delta-NARX model has been suggested for modeling nonlinear control systems, and it has been applied to the identification of a van der Pol oscillator (Anderson and Kadirkamanathan, 2007).

The theory of time scales is, however, not unique, and two approaches are followed in the literature: one dealing with the delta calculus (the forward approach); the other dealing with the nabla calculus (the backward approach) (Anderson et al., 2003). Available results on linear control systems on time scales are essentially restricted to the forward approach, but recent applications in economics have suggested that the backwards framework is sometimes more natural and preferable (Atici et al., 2006; Atici and Uvsal, 2008; Jackson, 2008; Almeida and Torres, 2009; Martins and Torres, 2009). This becomes evident when one considers that the time scales analysis can also have important implications for numerical analysts, who often prefer backward differences rather than forward differences to handle their computations due to practical implementation reasons and also for better stability properties of implicit discretizations.

The goal of this paper is to develop the foundations of a backward linear control systems theory on an arbitrary time scale. For that we make use of the recent duality theory (Caputo, 2009), which presents tools for obtaining nabla results from the delta calculus and viceversa, without making any assumptions on the regularity of the time scales (thus diverging from the approach in (Gürses et al., 2005)). The organization of the paper is as follows. Section 2 presents the main definitions and concepts of duality on time scales. In Section 3 we prove existence and uniqueness of a backward solution for time-varying linear control systems. In sections 4 and 5 we show that controllability and observability rank conditions are still valid for time-invariant and time-varying linear control systems defined on backward (dual) time scales. Finally, in Section 6 we prove conditions of existence of minimal realizations for the considered backward systems. We end with Section 7 of conclusions.

\section{Duality}

We assume the reader to be familiar with the calculus on time scales (Aulbach and Hilger, 1990; Bohner and Peterson, 2001). Let $\mathbb{T}$ be an arbitrary time scale and let $\mathbb{T}^{\star}:=\{s \in \mathbb{R}:-s \in \mathbb{T}\}$. Note that $\mathbb{T}^{\star}$ is a nonempty closed subset of the real line (so it is also a time scale), and that the map $\xi: \mathbb{T} \rightarrow \mathbb{T}^{*}$ defined by $\xi(t)=-t$ is onto and one-to-one. The new time scale $\mathbb{T}^{\star}$ is called the dual time scale (to $\mathbb{T}$ ). It follows that $\left(\mathbb{T}^{\kappa}\right)^{\star}=\left(\mathbb{T}^{\star}\right)_{\kappa}$ and $\left(\mathbb{T}_{\kappa}\right)^{\star}=\left(\mathbb{T}^{\star}\right)^{\kappa}$. By $[a, b]_{\mathbb{T}}$ we denote the intersection of the real interval $[a, b]$ with the time scale $\mathbb{T}$, i.e., $[a, b]_{\mathbb{T}}:=[a, b] \cap \mathbb{T}$. Similarly for $\mathbb{T}^{\star}$.

The dual function to $f: \mathbb{T} \rightarrow \mathbb{R}$, defined on $\mathbb{T}^{\star}$, is the function $f^{\star}: \mathbb{T}^{\star} \rightarrow \mathbb{R}$ given by $f^{\star}(s):=$ $f(-s)$ for all $s \in \mathbb{T}^{\star}$. It can be shown that $f$ is rd-continuous (resp. ld-continuous) if and only if its dual function $f^{\star}$ is ld-continuous (resp. rd-continuous) (Caputo, 2009).

Given $\sigma, \rho: \mathbb{T} \rightarrow \mathbb{T}$, the jump operators of the time scale $\mathbb{T}$, then the jump operators for time 
scale $\mathbb{T}^{\star}, \hat{\sigma}, \hat{\rho}: \mathbb{T}^{\star} \rightarrow \mathbb{T}^{\star}$, are given by the following relations (Caputo, 2009):

$$
\begin{aligned}
& \hat{\sigma}(s)=-\rho(-s)=-\rho^{\star}(s), \\
& \hat{\rho}(s)=-\sigma(-s)=-\sigma^{\star}(s),
\end{aligned}
$$

for all $s \in \mathbb{T}^{\star}$. These two equalities implies that the forward graininess $\mu: \mathbb{T} \rightarrow[0, \infty)$ and the backward graininess $\hat{\nu}: \mathbb{T}^{\star} \rightarrow[0, \infty)$ are related by

$$
\hat{\nu}(s)=\mu^{\star}(s) \quad \text { for all } s \in \mathbb{T}^{\star} .
$$

Similarly, the backward graininess $\nu: \mathbb{T} \rightarrow[0, \infty)$ and the forward graininess $\hat{\mu}: \mathbb{T}^{\star} \rightarrow[0, \infty)$ are related by

$$
\hat{\mu}(s)=\nu^{\star}(s) \quad \text { for all } s \in \mathbb{T}^{\star} .
$$

Lemma 2.1. (Caputo, 2009) Let function $f: \mathbb{T} \rightarrow \mathbb{R}$ be delta (resp. nabla) differentiable at point $t_{0} \in \mathbb{T}^{\kappa}$ (resp. at $t_{0} \in \mathbb{T}_{\kappa}$ ). Then function $f^{\star}: \mathbb{T}^{\star} \rightarrow \mathbb{R}$ is nabla (resp. delta) differentiable at $-t_{0} \in\left(\mathbb{T}^{\star}\right)_{\kappa}$ (resp. at $\left.-t_{0} \in\left(\mathbb{T}^{\star}\right)^{\kappa}\right)$ and the following relations hold true:

$$
f^{\Delta}\left(t_{0}\right)=-\left(f^{\star}\right)^{\hat{\nabla}}\left(-t_{0}\right) \quad\left(\text { resp. } \quad f^{\nabla}\left(t_{0}\right)=-\left(f^{\star}\right)^{\hat{\Delta}}\left(-t_{0}\right)\right),
$$

or

$$
f^{\Delta}\left(t_{0}\right)=-\left(\left(f^{\star}\right)^{\hat{\nabla}}\right)^{\star}\left(t_{0}\right) \quad\left(\operatorname{resp} . \quad f^{\nabla}\left(t_{0}\right)=-\left(\left(f^{\star}\right)^{\hat{\Delta}}\right)^{\star}\left(t_{0}\right)\right),
$$

or

$$
\left(f^{\Delta}\right)^{\star}\left(-t_{0}\right)=-\left(\left(f^{\star}\right)^{\hat{\nabla}}\right)\left(-t_{0}\right) \quad\left(\operatorname{resp} . \quad\left(f^{\nabla}\right)^{\star}\left(-t_{0}\right)=-\left(f^{\star}\right)^{\hat{\Delta}}\left(-t_{0}\right)\right) .
$$

Additionally, from properties of the $\Delta$ derivative on the time scale $\mathbb{T}$ and from (2), it follows that for any nabla differentiable function $f: \mathbb{T} \rightarrow \mathbb{R}$ its dual function $f^{\star}: \mathbb{T}^{\star} \rightarrow \mathbb{R}$ is delta differentiable with

$$
\left(f^{\star}\right)^{\hat{\sigma}}(s)=f^{\star}(s)+\hat{\mu}(s) \cdot\left(f^{\star}\right)^{\hat{\Delta}}(s) \quad \text { for all } s \in\left(\mathbb{T}^{\star}\right)^{\kappa} .
$$

Thus, $f^{\rho}(t)=f(t)-\nu(t) \cdot f^{\nabla}(t)$ for all $t \in \mathbb{T}_{\kappa}$.

Proposition 2.2. (Caputo, 2009)

(i) Let $f:[a, b]_{\mathbb{T}} \rightarrow \mathbb{R}$ be a rd-continuous function. Then,

$$
\int_{a}^{b} f(t) \Delta t=\int_{-b}^{-a} f^{\star}(s) \hat{\nabla} s .
$$

(ii) Let $f:[a, b]_{\mathbb{T}} \rightarrow \mathbb{R}$ be a ld-continuous function. Then,

$$
\int_{a}^{b} f(t) \nabla t=\int_{-b}^{-a} f^{\star}(s) \hat{\Delta} s .
$$

\section{Linear control systems}

Let us consider a time-varying system defined on a given time scale $\mathbb{T}$ :

$$
x^{\Delta}(t)=A(t) x(t)
$$

with $t \in \mathbb{T}^{\kappa}, t \geq t_{0}, t_{0} \in \mathbb{T}, A(t) \in \mathbb{R}^{n \times n}$. Recall that by transition function one means the unique forward solution of the system (3) with initial condition $x\left(t_{0}\right)=I$, where $I$ denotes the identity matrix $I \in \mathbb{R}^{n \times n}$. Its value at point $t \in \mathbb{T}$ is denoted by $\Phi_{A}\left(t, t_{0}\right)$. When $A$ is time invariant, we denote the solution of (3) with initial condition $x\left(t_{0}\right)=I$ by $e_{A}\left(t, t_{0}\right)$, and call it the exponential matrix function. There are important distinctions between the two notations, as $\Phi_{A}\left(t, t_{0}\right)=e_{A}\left(t, t_{0}\right)$ if and only if $A$ is a constant matrix.

By the backward system (to the system (3)) we mean

$$
y^{\hat{\nabla}}(s)=\bar{A}(s) y(s),
$$

defined on the dual time scale $\mathbb{T}^{\star}$ with $s \leq s_{0}=-t_{0}, s_{0} \in \mathbb{T}^{\star}, s \in\left(\mathbb{T}^{\star}\right)_{\kappa}$, and $\bar{A}(s):=-A^{\star}(s)$. 
Proposition 3.1. Let $\mathbb{T}^{\star}$ be a time scale. Then the time-varying system

$$
y^{\hat{\nabla}}(s)=\bar{A}(s) y(s), \quad y\left(s_{0}\right)=y_{0},
$$

where $s_{0} \in \mathbb{T}^{\star}, s \in\left(\mathbb{T}^{\star}\right)_{\kappa}$, and $\bar{A} \in \mathbb{R}^{n \times n}$, has a unique backward solution. This solution is of the form $y(s)=\Psi_{\bar{A}}\left(s, s_{0}\right) y_{0}$ for any $s \leq s_{0}$, where $\Psi$ denotes the transition function dual to $\Phi$, i.e., $\Psi_{\bar{A}}\left(s, s_{0}\right)=\left(\Phi_{-\bar{A}^{\star}}\right)^{\star}\left(-s,-s_{0}\right)$.

Proof. Let $t_{0}:=-s_{0}$ and $t:=-s$ for any $s \in\left(\mathbb{T}^{\star}\right)_{\kappa}$. From Lemma 2.1 it follows that the system (3) defined on $\mathbb{T}$ (the dual of $\mathbb{T}^{\star}$ ) with initial condition $x\left(t_{0}\right)=y_{0}$ can be rewritten on $\mathbb{T}^{\star}$ as

$$
y^{\hat{\nabla}}(s)=-A^{\star}(s) y(s), \quad y\left(s_{0}\right)=y_{0},
$$

where $y$ is the dual vector function to $x$, i.e., $y(s)=x^{\star}(-s)$. Because system (3) with initial condition $x\left(t_{0}\right)=y_{0}$ has a unique forward solution on $\mathbb{T}$ (Bohner and Peterson, 2001; Jackson, 2007), then system (5) has also a unique backward solution $y(s)=\Psi_{-A^{\star}}\left(s, s_{0}\right) y_{0}$ on $\mathbb{T}^{\star}$.

It follows from Proposition 3.1 that the solutions of (3) and (4) are related by $x(t)=y^{\star}(-t)$ and $y(s)=x^{\star}(-s)$.

Remark 1. In the case of a time-invariant system (3), i.e., when $A$ is a constant matrix, we have $e_{A}\left(t, t_{0}\right)=\left(\hat{e}_{\bar{A}}\right)^{\star}\left(-t,-t_{0}\right)$ with $\hat{e}_{\bar{A}}$ the nabla exponential function on $\mathbb{T}^{\star}$ for the constant matrix $\bar{A}=-A^{\star}$.

Let us consider now a time-varying control system on the time scale $\mathbb{T}$ :

$$
x^{\Delta}(t)=A(t) x(t)+B(t) u(t), \quad x\left(t_{0}\right)=x_{0},
$$

where $u(\cdot)$ is a rd-continuous control taking values in $\mathbb{R}^{m}$, and $A(t) \in \mathbb{R}^{n \times n}$ and $B(t) \in \mathbb{R}^{n \times m}$ are time-dependent matrices defined on $\mathbb{T}^{\kappa}$.

Proposition 3.2. Let $\mathbb{T}^{\star}$ be the dual time scale to $\mathbb{T}$, with $\mathbb{T}$ the time scale where the control system (6) is defined. Then the control system

$$
y^{\hat{\nabla}}(s)=\bar{A}(s) y(s)+\bar{B}(s) v(s), \quad y\left(s_{0}\right)=x_{0},
$$

where $s_{0}=-t_{0}, s \leq s_{0}, s \in\left(\mathbb{T}^{\star}\right)_{\kappa}, \bar{A}(s)=-A^{\star}(s)$, and $\bar{B}(s)=-B^{\star}(s)$, has a unique backward solution. The solution is given by

$$
y(s)=\Psi_{\bar{A}}\left(s, s_{0}\right) x_{0}+\int_{s}^{s_{0}} \Psi_{\bar{A}}(s,-\hat{\rho}(\varsigma)) \bar{B}(\varsigma) v(\varsigma) \hat{\nabla} \varsigma
$$

for any $s \leq s_{0}$, where $\Psi$ is the transition function dual to $\Phi$, i.e., $\Psi_{\bar{A}}\left(s, s_{0}\right)=\left(\Phi_{A}\right)^{\star}\left(-s,-s_{0}\right)$.

Proof. Let $s:=-t$ for any $t \in \mathbb{T}^{\kappa}$. So $s \in\left(\mathbb{T}^{\star}\right)_{\kappa}$ and $s \leq s_{0}$. The control system (6) defined on $\mathbb{T}$ can be rewritten on the time scale $\mathbb{T}^{\star}$ as

$$
y^{\hat{\nabla}}(s)=\bar{A}(s) y(s)+\bar{B}(s) v(s),
$$

where $y$ and $v$ are the dual vector to $x$ and $u$, respectively, i.e., $y(s)=x^{\star}(-s)$ and $v(s)=u^{\star}(-s)$. Equation (6) has a unique forward solution on $\mathbb{T}$ (Bohner and Peterson, 2001; Jackson, 2007) given by

$$
x(t)=\Phi_{A}\left(t, t_{0}\right) x_{0}+\int_{t_{0}}^{t} \Phi_{A}(t, \sigma(\tau)) B(\tau) u(\tau) \Delta \tau .
$$

Thus, by definition of dual time scale and by (1), the dual control system (8) on $\mathbb{T}^{*}$ has also a unique, but backward, solution

$$
y(s)=\Psi_{-A^{\star}}\left(s, s_{0}\right) x_{0}-\int_{s}^{s_{0}} \Psi_{-A^{\star}}(s,-\hat{\rho}(\varsigma)) B^{\star}(\varsigma) u^{\star}(\varsigma) \hat{\nabla} \varsigma .
$$


It follows from Proposition 3.2 that state and control variables of systems (6) and (77) are related by $(x(t), u(t))=\left(y^{\star}(-t), v^{\star}(-t)\right)$ and $(y(s), v(s))=\left(x^{\star}(-s), u^{\star}(-s)\right)$.

Remark 2. In the particular case when $\bar{A}$ and $\bar{B}$ are constant matrices, then the solution of (7) given in Proposition 3.2 takes the form

$$
y(s)=\hat{e}_{\bar{A}}\left(s, s_{0}\right) x_{0}+\int_{s}^{s_{0}} \hat{e}_{\bar{A}}(s,-\hat{\rho}(\varsigma)) \bar{B} v(\varsigma) \hat{\nabla} \varsigma
$$

for any $s \leq s_{0}$.

\section{Controllability}

Let $\mathbb{T}^{\star}$ be a given time scale with operators $\hat{\sigma}, \hat{\rho}, \hat{\mu}, \hat{\nu}, \hat{\Delta}$, and $\hat{\nabla}$. Let us consider the following linear time-varying system defined on $\mathbb{T}^{\star}$ :

$$
\begin{aligned}
\bar{\Lambda}: \quad y^{\hat{\nabla}}(s) & =\bar{A}(s) y(s)+\bar{B}(s) v(s) \\
\gamma(s) & =\bar{C}(s) y(s)+\bar{D}(s) v(s)
\end{aligned}
$$

with initial condition $y\left(s_{0}\right)=y_{0}, s \leq s_{0}$, where $y(s) \in \mathbb{R}^{n}$ is the state of the system at time $s$, $v(s) \in \mathbb{R}^{m}$ is the control value at time $s$, and $\bar{A}(s) \in \mathbb{R}^{n \times n}, \bar{B}(s) \in \mathbb{R}^{n \times m}, \bar{C}(s) \in \mathbb{R}^{p \times n}$, and $\bar{D}(s) \in \mathbb{R}^{p \times m}, p, m \leq n$, are ld-continuous as functions of $s$.

We say that system $\bar{\Lambda}$ is controllable if for any two states $y_{0} \in \mathbb{R}^{n}$ and $y_{1} \in \mathbb{R}^{n}$ there exist $s_{0}, s_{1} \in \mathbb{T}^{\star}, s_{1}<s_{0}$, and a piecewise ld-continuous control $v(s), s \in\left[s_{1}, s_{0}\right]_{\mathbb{T}^{\star}}$, such that for $y_{0}=y\left(s_{0}\right)$ one has $y\left(s_{1}\right)=y_{1}$. The set of all points that can be reachable from the point $y_{0}=y\left(s_{0}\right)$ in time $s_{1} \in \mathbb{T}^{\star}$ is called the reachable set from $y_{0}$ in time $s_{1}$ and is denoted by $\overline{\mathcal{R}}_{y_{0}}\left(s_{1}, s_{0}\right)$. The set of all points reachable from $y_{0}=y\left(s_{0}\right)$ in finite time $s \in \mathbb{T}^{\star}$ will be denoted by $\overline{\mathcal{R}}_{y_{0}}\left(s_{0}\right)$. Note that $\overline{\mathcal{R}}_{y_{0}}\left(s_{1}, s_{0}\right)=\Psi_{\bar{A}}\left(s_{1}, s_{0}\right)+\overline{\mathcal{R}}_{0}\left(s_{1}, s_{0}\right)$.

Let us assume that $\mathbb{T}^{\star}$ is a time scale for which $\hat{\rho}$ is sufficiently ld-continuous $\hat{\nabla}$ differentiable. Define the sequence of matrices

$$
\bar{K}_{j}(s):=-\left.\frac{\partial^{j}}{\hat{\nabla} z^{j}}\left[\Psi_{\bar{A}}(\hat{\rho}(s),-\hat{\rho}(z)) \bar{B}(z)\right]\right|_{z=s} \quad j=0,1,2, \ldots
$$

Theorem 4.1. Let $r$ be a positive integer such that $\bar{B}(\cdot)$ is $r$-times ld-continuously $\hat{\nabla}$ differentiable and both $\hat{\rho}(\cdot)$ and $\bar{A}(\cdot)$ are ld-continuously $r-1$-times $\hat{\nabla}$ differentiable on $\left[s_{0}, s_{1}\right]_{\mathbb{T}^{*}}$. Then the linear system $\bar{\Lambda}$ is controllable on $\left[s_{1}, s_{0}\right]_{\mathbb{T} *}$ if for some $s_{c} \in\left(s_{1}, s_{0}\right]_{\mathbb{T} *}$ the matrix

$$
\left(\bar{K}_{0}\left(s_{c}\right) \bar{K}_{1}\left(s_{c}\right) \ldots \bar{K}_{r}\left(s_{c}\right)\right)
$$

is of full rank, where $\bar{K}_{j}, j=0,1, \ldots, r$, are the matrices given by (9).

Proof. Let $t:=-s$ (and $t_{0}:=-s_{0}$ ) so that $t$ belongs to $\mathbb{T}-$ the dual time scale of $\mathbb{T}^{\star}-$ with operators $\sigma, \rho, \mu, \nu, \Delta$, and $\nabla$. Using Lemma 2.1 we can rewrite system $\bar{\Lambda}$ on $\mathbb{T}$ as

$$
\begin{aligned}
\Lambda: \quad x^{\Delta}(t) & =A(t) x(t)+B(t) u(t), \quad x\left(t_{0}\right)=y_{0}, \\
z(t) & =C(t) x(t)+D(t) u(t),
\end{aligned}
$$

where $A(t) \in \mathbb{R}^{n \times n}, B(t) \in \mathbb{R}^{n \times m}, C(t) \in \mathbb{R}^{p \times n}$, and $D(t) \in \mathbb{R}^{p \times m}, p, m \leq n$, are rd-continuous as functions of $t$. System $\Lambda$ is controllable on an interval $\left[t_{0}, t_{1}\right]_{\mathbb{T}}$ if for some $t_{c} \in\left[t_{0}, t_{1}\right)_{\mathbb{T}}$

$$
\operatorname{rank}\left(K_{0}\left(t_{c}\right) K_{1}\left(t_{c}\right) \ldots K_{r}\left(t_{c}\right)\right)=n
$$

with matrices $K_{j}(s):=-\frac{\partial^{j}}{\Delta^{j}}\left[\Phi_{A}(\sigma(t), \sigma(s)) B(s)\right]_{\left.\right|_{s=t}}, j=0,1, \ldots, r$. This fact was proved in (Davis et al., 2009) for a regressive system $\Lambda$. The proof is still valid without the regressivity 
assumption. The dual system to $\Lambda$ is $\bar{\Lambda}$ where $y$ is the dual vector to $x, \bar{A}(s):=-A^{\star}(s), \bar{B}(s):=$ $-B^{\star}(s), \bar{C}(s):=C^{\star}(s), \bar{D}(s):=D^{\star}(s)$ and $A^{\star}, B^{\star}, C^{\star}$, and $D^{\star}$ are the dual function matrices to $A, B, C$ and $D$, respectively. Moreover, from the form of matrices $K_{j}$ and Lemma 2.1, it follows that the dual matrix $K^{\star}$ is of form

$$
K_{j}^{\star}(-t)=-\left.\frac{\partial^{j}}{\hat{\nabla} s^{j}}\left[\Phi_{-A^{*}}^{*}(\hat{\rho}(s),-\hat{\rho}(z)) B^{\star}(z)\right]\right|_{z=s},
$$

where $\Phi_{-A^{*}}^{\star}$ is the dual matrix to $\Phi_{A}$. We obtain (9) by putting $\bar{K}=K^{\star}$ and $\Psi_{\bar{A}}=\Phi_{-A^{*}}^{\star}$.

Example 4.2. Let us consider the control system

$$
\left\{\begin{array}{l}
y_{1}^{\hat{\nabla}}=y_{1}+s^{2} y_{2}+v_{1} \\
y_{2}^{\hat{\nabla}}=-y_{2}-s v_{2}
\end{array}\right.
$$

defined on the time scale $\mathbb{T}^{\star}$ dual of $\mathbb{T}=\bigcup_{k \in \mathbb{Z}}[2 k, 2 k+1]$. Because function

$$
\Psi_{\bar{A}}=\left[\begin{array}{cc}
2^{k-l} \mathrm{e}^{s-z} & 2^{k-l} s^{2}(s-z) \mathrm{e}^{s-z}+2^{k-l-1} s^{2}(k-l) \mathrm{e}^{s-z} \\
0 & 2^{k-l} \mathrm{e}^{s-z}
\end{array}\right]
$$

is the transition matrix of this system, then

$$
\begin{aligned}
& K_{0}=\left(\begin{array}{c}
1 \\
-s
\end{array}\right), \\
& K_{1}=-\left.\frac{\partial}{\hat{\nabla} z}\left[\begin{array}{c}
2^{k-l} \mathrm{e}^{s-z}-\left(2^{k-l} s^{2}(s-z) \mathrm{e}^{s-z}+2^{k-l-1} s^{2}(k-l) \mathrm{e}^{s-z}\right) s \\
-s 2^{k-l} \mathrm{e}^{s-z}
\end{array}\right]\right|_{z=s} .
\end{aligned}
$$

For any $s \in(2 l-1, z], s \neq 0, \operatorname{rank}\left(\begin{array}{cc}K_{0} & K_{1}\end{array}\right)=2$. If $s=2 l-1$, then for $z \in(2 l-3,2 l-2]$ $\operatorname{rank}\left(\begin{array}{ll}K_{0} & K_{1}\end{array}\right)=2 \Leftrightarrow s \neq 0$. We conclude that system (11) is controllable on $\mathbb{R}^{2} \backslash\{0\}$.

Let us consider now a time-invariant system:

$$
\begin{aligned}
y^{\hat{\nabla}}(s) & =\bar{A} y(s)+\bar{B} v(s) \\
\gamma(s) & =\bar{C} y(s)
\end{aligned}
$$

with initial condition $y\left(s_{0}\right)=y_{0}, s \leq s_{0}$, and $\bar{A} \in \mathbb{R}^{n \times n}, \bar{B} \in \mathbb{R}^{n \times m}$ and $\bar{C} \in \mathbb{R}^{p \times n}$ constant matrices.

Theorem 4.3. Let us assume that the interval $\left[s_{1}, s_{0}\right]_{\mathbb{T}^{*}}$ consists of at least $n+1$ elements. The following conditions are equivalent:

(i) $\overline{\mathcal{R}}_{y_{0}}\left(s_{1}, s_{0}\right)=\mathbb{R}^{n}$;

(ii) $\operatorname{rank}\left(\bar{P}_{0} \bar{B}, \bar{P}_{1} \bar{B}, \ldots, \bar{P}_{n-1} \bar{B}\right)=n$ where matrices $\bar{P}_{i}, i=0,1, \ldots, n-1$, are given recursively by $\bar{P}_{0}=I$ and $\bar{P}_{k+1}=\left(\bar{A}-\lambda_{k+1} I\right) \bar{P}_{k}, k=0,1, \ldots, n-1$, with $\lambda_{1}, \ldots, \lambda_{n}$ the eigenvalues of the matrix $\bar{A}$;

(iii) $\operatorname{rank}\left(\bar{B}, \bar{A} \bar{B}, \ldots, \bar{A}^{n-1} \bar{B}\right)=n$;

(iv) the system (12) is controllable.

Proof. Let $t:=-s$ for any $s \in \mathbb{T}^{\star}$ and let $t_{0}:=-s_{0}$, so that $t$ is an element of the dual time scale $\mathbb{T}$ (with operators $\sigma, \rho, \mu, \nu, \Delta, \nabla$ ). Using Lemma 2.1 we write the dual system on $\mathbb{T}$ :

$$
\begin{aligned}
x^{\Delta}(t) & =A x(t)+B u(t), \\
z(t) & =C x(t), \\
x\left(t_{0}\right) & =y_{0}
\end{aligned}
$$

with $A=-\bar{A}, B=-\bar{B}$, and $C=\bar{C}$. Since conditions 
(a) $\mathcal{R}_{y_{0}}\left(t_{0}, t_{1}\right)=\mathbb{R}^{n}$;

(b) $\operatorname{rank}\left(P_{0} B, P_{1} B, \ldots, P_{n-1} B\right)=n$ where matrices $P_{i}, i=0,1, \ldots, n-1$, are given recursively by $P_{0}=I$ and $P_{k+1}=\left(A-\lambda_{k+1} I\right) P_{k}, k=0,1, \ldots, n-1$, with $\lambda_{1}, \ldots, \lambda_{n}$ the eigenvalues of the matrix $A$;

(c) $\operatorname{rank}\left(B, A B, \ldots, A^{n-1} B\right)=n$;

(d) the system given by (13) is controllable;

are equivalent on the time scale $\mathbb{T}$ (for the proof see (Bartosiewicz and Pawluszewicz, 2006)), then, after coming back to the time scale $\mathbb{T}^{\star}$ we see that conditions $(i)-(i v)$ are also equivalent on the time scale $\mathbb{T}^{\star}$.

Example 4.4. Let us consider the control system

$$
\left\{\begin{array}{l}
y_{1}^{\hat{\nabla}}=y_{2} \\
y_{2}^{\hat{\nabla}}=-3 y_{1}-4 y_{2}+v .
\end{array}\right.
$$

Independently of the time scale, we have always $\operatorname{rank}(\bar{B}, \bar{A} \bar{B})=2$. Thus the system (14) is controllable by items (iii) and (iv) of Theorem 4.3.

\section{Observability}

Let us consider the linear time-varying control system

$$
\begin{aligned}
\bar{\Lambda}: \quad y^{\hat{\nabla}}(s) & =\bar{A}(s) y(s)+\bar{B}(s) v(s) \\
\gamma(s) & =\bar{C}(s) y(s),
\end{aligned}
$$

$s \leq s_{0}$, defined on a given time scale $\mathbb{T}^{\star}$ under a given initial condition $y\left(s_{0}\right)=y_{0}$. We say that such a system $\bar{\Lambda}$ is observable on $\left[s_{1}, s_{0}\right]_{\mathbb{T}^{*}}$ if any initial state $y\left(s_{0}\right)=y_{0}$ can be uniquely determined by the output function $\gamma(s)$ for $s \in\left(s_{1}, s_{0}\right]_{\mathbb{T}^{\star}}$.

Let us assume that the time scale $\mathbb{T}^{\star}$ is such that $\hat{\rho}$ is sufficiently $\hat{\nabla}$ differentiable with ldcontinuous derivatives. Define the sequence of matrices

$$
\bar{L}_{j}(s):=-\left.\frac{\partial^{j}}{\hat{\nabla} z^{j}}\left[\bar{C}(s) \Psi_{\bar{A}}(s, z)\right]\right|_{z=s}, \quad j=0,1,2, \ldots
$$

Using a similar reasoning as in Theorem 4.1 we have the following:

Theorem 5.1. Let $r$ be a positive integer such that the matrix function $\bar{C}(s)$ is $r$-times ldcontinuously $\hat{\nabla}$ differentiable and both $\hat{\rho}(s)$ and $\bar{A}(s)$ are $r$-1-times ld-continuously $\hat{\nabla}$ differentiable for any $s \in\left[s_{0}, s_{1}\right]_{\mathbb{T}^{\star}}$. Then the linear system (15) is observable on $\left[s_{1}, s_{0}\right]_{\mathbb{T}^{*}}$ if for some $s_{c} \in\left(s_{1}, s_{0}\right]_{\mathbb{T}^{\star}}$ the matrix

$$
\left(\begin{array}{c}
\bar{L}_{0}\left(s_{c}\right) \\
\bar{L}_{1}\left(s_{c}\right) \\
\vdots \\
\bar{L}_{r}\left(s_{c}\right)
\end{array}\right)
$$

is of full rank, where $\bar{L}_{j}$ are the matrices given by (16), $j=0,1, \ldots, r$.

Let us consider the particular case of a time invariant system (12) on the time scale $\mathbb{T}^{\star}$ :

Theorem 5.2. Assume that the interval $\left[s_{1}, s_{0}\right]_{\mathbb{T} *}$ consists of at least $n+1$ points. The following conditions are equivalent:

(i) system (12) is observable; 
(ii) rank $\left(\begin{array}{c}\bar{C} \bar{P}_{0} \\ \bar{C} \bar{P}_{1} \\ \vdots \\ \bar{C} \bar{P}_{n-1}\end{array}\right)=n$, where matrices $\bar{P}_{i}, i=0,1, \ldots, n-1$, are given recursively by $\bar{P}_{0}=I$, $\bar{P}_{k+1}=\left(\bar{A}-\lambda_{k+1} I\right) \bar{P}_{k}, k=0, \ldots, n-1$, and $\lambda_{1}, \ldots, \lambda_{n}$ are the eigenvalues of $\bar{A} ;$

(iii) $\operatorname{rank}\left(\begin{array}{c}\bar{C} \\ \bar{C} \bar{A} \\ \vdots \\ \bar{C} \bar{A}^{n-1}\end{array}\right)=n$.

Proof. Without loss of generality we may assume that $\bar{B}(s)=0$. Putting $t:=-s$ for any $s \in \mathbb{T}^{\star}$ and letting $t_{0}:=-s_{0}$, we rewrite the system from the time scale $\mathbb{T}^{\star}$ onto $\mathbb{T}$ : using Lemma 2.1 we can rewrite the given system (12) in the form (13). Since conditions,

(a) system (13) is observable;

(b) $\operatorname{rank}\left(\begin{array}{c}C P_{0} \\ C P_{1} \\ \vdots \\ C P_{n-1}\end{array}\right)=n$, where matrices $P_{i}, i=0, \ldots, n-1$, are given recursively by $P_{0}=I$ and $P_{k+1}=\left(A-\lambda_{k+1} I\right) P_{k}, k=0, \ldots, n-1$, with $\lambda_{1}, \ldots, \lambda_{n}$ the eigenvalues of $A$;

(c) $\operatorname{rank}\left(\begin{array}{c}C \\ C A \\ \vdots \\ C A^{n-1}\end{array}\right)=n$;

are equivalent on the time scale $\mathbb{T}$ (see (Bartosiewicz and Pawłuszewicz, 2006)), taking $s_{0}:=-t_{0}$ and $s:=-t$ for any $t \in \mathbb{T}$ we obtain equivalence of conditions $(i)-(i i i)$ on the time scale $\mathbb{T}^{\star}$.

\section{Realizability}

One can notice that the control $v(\cdot)$ and the output $\gamma(\cdot)$ of system $\bar{\Lambda}$ given by (15) are related in the following way:

$$
\gamma(s)=C(s) \Psi_{\bar{A}}\left(s, s_{0}\right) y_{0}+\int_{s}^{s_{0}} C(s) \Psi_{\bar{A}}(s,-\hat{\rho}(z)) \bar{B}(z) v(z) \hat{\nabla} z
$$

for $s \leq s_{0}, s_{0} \in \mathbb{T}^{\star}$ fixed. The operator $\overline{\mathcal{A}}[v(\cdot)]:=\int_{s}^{s_{0}} \bar{G}(s, z) v(z) \hat{\nabla} z$, where

$$
\bar{G}(s, z)=C(s) \Psi_{\bar{A}}(s,-\hat{\rho}(z)) \bar{B}(z),
$$

is called the action of $\bar{\Lambda}$, while function $\bar{G}$ is called the weighting pattern of the system. Note that different systems of form (15) can define the same weighting pattern. Each of them is called a realization. A realization is minimal if no realization of $\bar{G}(s, z)$ with dimension less than $n$ exists ( $n$ is the dimension of matrix $\bar{A}$ ).

Theorem 6.1. The weighting pattern $\bar{G}(s, z)$ is realizable if and only if for each $s_{1} \in \mathbb{T}^{\star}, s_{1} \leq s_{0}$, there exists a ld-continuous matrix function $\bar{H}:\left(-\infty, s_{1}\right]_{\mathbb{T}^{*}} \rightarrow \mathbb{R}^{q \times n}$ and a ld-continuous matrix function $\bar{F}:\left(-\infty, s_{1}\right]_{\mathbb{T}^{\star}} \rightarrow \mathbb{R}^{n \times r}$ such that

$$
\bar{G}(s, z)=\bar{H}(s) \bar{F}(z) .
$$


Proof. Taking $t:=-s$ and $\tau=-z$ (so that $t, \tau \in \mathbb{T}$, where $\mathbb{T}$ is the dual time scale of $\mathbb{T}^{\star}$ with operators $\sigma, \rho, \mu, \nu, \Delta$, and $\nabla$ ) and using Lemma 2.1, we can rewrite system $\bar{\Lambda}$ (15) into the system $\Lambda(10)$ on the time scale $\mathbb{T}$. A forward characterization of realizable systems on $\mathbb{T}$ can be obtained from (Davis et al., 2009). Note that if $G^{\star}$ is the dual function to $G$ and $H^{\star}, F^{\star}$ are dual to $H$ and $F$, respectively (note that $H^{\star}$ and $F^{\star}$ are ld-continuous), then $G^{\star}(-t,-\sigma(-\tau))=$ $H^{\star}(-t) F^{\star}(-\sigma(-t \tau)$. On the other hand, we have the weighting pattern

$$
G^{\star}(-t,-\sigma(-\tau))=C^{\star}(-t) \Phi_{-A^{\star}}(-t,-\sigma(-\tau))\left(-B^{\star}(\tau)\right) .
$$

It means that for $\bar{G}=G^{\star}, \bar{H}=H^{\star}$, and $\bar{F}=F^{\star}$, the weighting pattern $\bar{G}(s,-\hat{\rho}(z))$ is realizable if and only if $\bar{G}(s,-\hat{\rho}(z))=\bar{H}(s) \bar{F}(-\hat{\rho}(z))$, where $\bar{G}(s,-\hat{\rho}(z))=C(s) \Psi_{\bar{A}}(s,-\hat{\rho}(z)) \bar{B}(z)$.

Remark 3. For time-invariant systems the weighting pattern is given by $\bar{G}(s,-\hat{\rho}(z))=C \Psi_{\bar{A}}(s,-\hat{\rho}(z)) \bar{B}$. The backward characterization of realizable time-invariant systems on time scales follows from Theorem 6.1, and the forward characterization of realizable time-invariant systems on time scales is given in (Bartosiewicz and Pawtuszewica, 2006).

Definition 6.2. We say that control system $\bar{\Lambda}$ given by (15) is progressive if matrix $I-\hat{\nu}(s) \bar{A}(s)$ is invertible.

Remark 4. If $\bar{\Lambda}$ is progressive, then its dual $\bar{\Lambda}^{\star}=\Lambda$ is regressive - see (Bohner and Peterson, 2001) for the definition of regressivity.

Theorem 6.3. Let us assume that the control system $\bar{\Lambda}$ given by (15) is progressive. Let $\bar{\Lambda}$ be a realization of the weighting pattern $\bar{G}(s,-\hat{\rho}(z))$. Then this realization is minimal if and only if for some $s_{0}$ and $s_{1}<s_{0}$ the system $\bar{\Lambda}$ is both controllable and observable on $\left[s_{1}, s_{0}\right]_{\mathbb{T}^{*}}$.

Proof. Taking $t:=-s, \tau=-z$, and $x_{0}=y_{0}^{\star}$ (so that $t, \tau \in \mathbb{T}$ where $\mathbb{T}$ is the dual time scale of $\mathbb{T}^{\star}$ with operators $\left.\sigma, \rho, \mu, \nu, \Delta, \nabla\right)$ and using Lemma 2.1 we can convert system $\bar{\Lambda}$ onto system $\Lambda$ defined on $\mathbb{T}$ :

$$
\begin{aligned}
\Lambda: \quad x^{\Delta}(t) & =A(t) x(t)+B(t) u(t), \\
\vartheta(t) & =C(t) x(t), \\
x\left(t_{0}\right) & =x_{0}
\end{aligned}
$$

where $A(t) \in \mathbb{R}^{n \times n}, B(t) \in \mathbb{R}^{n \times m}$, and $C(t) \in \mathbb{R}^{p \times n}, p, m \leq n$, are rd-continuous matricial functions of $t$. Under the regressivity assumption system $\Lambda$ is a realization of the weighting pattern $G(t, \sigma(\tau))$ if and only if for some $t_{0}$ and $t_{1}<t_{0}$ the system is both controllable and observable on $\left[t_{0}, t_{1}\right]_{\mathbb{T}}$ (Davis et al., 2009). Now, if $s:=-t, z:=-\tau$ and $s_{0}:=-t_{0}$ for $t, \tau, t_{0} \in \mathbb{T}$, then the dual system to $\Lambda$ is of the form of the system $\bar{\Lambda}$ with $y$ the dual vector to $x, \bar{A}(s):=-A^{\star}(s)$, $\bar{B}(s):=-B^{\star}(s)$, and $\bar{C}(s):=C^{\star}(s)$. Moreover, from (17) we can see that the weighting pattern on the time scale $\mathbb{T}^{\star}$ is exactly of the form $\bar{G}(s,-\hat{\rho}(z))$. Since this gives a one-to-one correspondence between the considered systems, the realization given by $\bar{G}(s,-\hat{\rho}(z))$ is a minimal one if and only if for some $s_{0}$ and $s_{1}<s_{0}$ the system $\bar{\Lambda}$ is both (backward) controllable and observable on $\left[s_{1}, s_{0}\right]_{\mathbb{T}^{*}}$.

For the time invariant case we can prove minimal realization without assuming progressivity.

Theorem 6.4. Let system (12) be a realization of the weighting pattern $\bar{G}(s,-\hat{\rho}(z))$. This realization is minimal if and only if for some $s_{0}$ and $s_{1}<s_{0}$ the system (12) is both controllable and observable on $\left[s_{1}, s_{0}\right]_{\mathbb{T}^{*}}$.

Proof. The result follows by applying Caputo's duality (Caputo, 2009) to the control system (12) and using Theorem 5.7 and Corollary 5.8 in (Bartosiewicz and Pawłuszewicz, 2006). 
Example 6.5. Let us consider a control system

$$
\left\{\begin{array}{l}
y_{1}^{\hat{\nabla}}=y_{1}-y_{2}+v_{1} \\
y_{2}^{\hat{\nabla}}=2 y_{2}+v_{2} \\
\gamma_{1}=y_{1} \\
\gamma_{2}=-y_{2}
\end{array}\right.
$$

defined on the time scale $\mathbb{T}^{\star}$ dual of $\mathbb{T}=\bigcup_{k \in \mathbb{Z}}[2 k, 2 k+1]$. It is easy to see that this system is both controllable and observable. Because

$$
\hat{e}_{\bar{A}}=\left(\begin{array}{cc}
2^{k-l} e^{s-z} & 2^{k-l} e^{s-z}-3^{k-l} e^{2(s-z)} \\
0 & 3^{k-l} e^{2(s-z)}
\end{array}\right)
$$

for $k \neq l$, and

$$
\hat{e}_{\bar{A}}=\left(\begin{array}{cc}
e^{s-z} & e^{s-z}-e^{2(s-z)} \\
0 & e^{2(s-z)}
\end{array}\right)
$$

for $k=l$, then for any $s_{1} \in[2 p-1,2 p]_{\mathbb{T}^{\star}}$ and $k<p<l$

$$
\bar{G}(s, z)=\left(\begin{array}{ll}
2^{k-p-s_{1}} e^{s-s_{1}} & -2 \cdot 3^{k-p-s_{1}} e^{2\left(s-s_{1}\right)}
\end{array}\right)\left(\begin{array}{c}
2^{-\left(k-p+s_{1}\right)} e^{-\left(z-s_{1}\right)} \\
3^{s_{1}} e^{-2\left(z-s_{1}\right)}
\end{array}\right) ;
$$

while for any $s_{1} \in[z, s]_{\mathbb{T}^{*}}$ and $k=l$

$$
\bar{G}(s,-\hat{\rho}(z))=\left(\begin{array}{ll}
2 e^{s-s_{1}} & -2 e^{2\left(s-s_{1}\right)}
\end{array}\right)\left(\begin{array}{c}
e^{-\left(z-s_{1}\right)} \\
e^{-2\left(z-s_{1}\right)}
\end{array}\right) .
$$

\section{Conclusions}

The calculus on time scales has been developed about 20 years ago by Hilger in order to unify various parallel results in the theory of discrete and continuous dynamical systems (Hilger, 1990). It found considerable number of applications over the last decade, particularly in the context of engineering applications and systems theory and control (DaCunha, 2005; Bartosiewicz et al., 2007; Jackson, 2007; Bartosiewicz and Pawluszewicz, 2008; Seiffertt et al., 2008; Davis et al., 2009; Kotta et al., 2009; Pawłuszewicz and Torres, 2010). The time scales calculus allows two dual formulations: the delta-calculus where the derivative is the forward difference operator (yielding the explicit Euler scheme) and the nabla-calculus with the backward difference operator as derivative (and the implicit Euler scheme) when the time scale $\mathbb{T}$ is $\mathbb{Z}$. The duality between the two approaches, on an arbitrary time scale $\mathbb{T}$, has been recently exploited (Caputo, 2009). Here we introduce the study of backward linear control systems defined on an arbitrary time scale. We claim that such systems are important in applications because they rely on information about past values of states and/or outputs. Indeed, as pointed out in (Jackson, 2008), the nabla time scales analysis has important implications for numerical analysts, who often use backward differences instead of forward differences in their computations. In this work controllability, observability, and realizability conditions for nabla time-varying linear control systems are proved using duality arguments and corresponding delta results. Illustrative examples are given. We trust that the approach here promoted can open further directions of future research.

\section{Acknowledgement}

The authors were partially supported by the Center for Research on Optimization and Control (CEOC) and by the Center of Research and Development in Mathematics and Applications (CIDMA) from the Portuguese Foundation for Science and Technology (FCT), cofinanced by the European Community fund FEDER/POCI 2010.

The authors would like to express their gratitude to two anonymous referees for relevant and stimulating remarks. 


\section{References}

Almeida, R., and Torres, D.F.M. (2009), "Isoperimetric problems on time scales with nabla derivatives," J. Vib. Control, 15, 951-958. arXiv:0811.3650

Anderson, D., Bullock, J., Erbe, L., Peterson, A., and Tran, H. (2003), "Nabla dynamic equations," in Advances in dynamic equations on time scales Boston, MA: Birkhäuser Boston, pp. 47-83.

Anderson, S., and Kadirkamanathan, V. (2007), "Modelling and identification of nonlinear deterministic systems in the delta-domain.," Automatica, 43, 1859-1868.

Atici, F.M., Biles, D.C., and Lebedinsky, A. (2006), "An application of time scales to economics," Math. Comput. Modelling, 43, 718-726.

Atici, F.M., and Uysal, F. (2008), "A production-inventory model of HMMS on time scales," Appl. Math. Lett., 21, 236-243.

Aulbach, B., and Hilger, S. (1990), "A unified approach to continuous and discrete dynamics," in Qualitative theory of differential equations (Szeged, 1988), Vol. 53 of Colloq. Math. Soc. János Bolyai, Amsterdam: North-Holland, pp. 37-56.

Bartosiewicz, Z., Kotta, Ü., and Pawłuszewicz, E. (2006), "Equivalence of linear control systems on time scales," Proc. Estonian Acad. Sci. Phys. Math., 55, 43-52.

Bartosiewicz, Z., and Pawłuszewicz, E. (2006), "Realizations of linear control systems on time scales," Control Cybernet., 35, 769-786.

Bartosiewicz, Z., and Pawluszewicz, E. (2008), "Realizations of nonlinear control systems on time scales," IEEE Trans. Automat. Control, 53, 571-575.

Bartosiewicz, Z., Piotrowska, E., , and Wyrwas, M. (2007), "Stability, stabilization and observers of linear control systems on time scales," in Proc. IEEE Conf. on Decision and Control, December, New Orleans, LA, pp. 2803-2808.

Bohner, M., and Peterson, A., Dynamic equations on time scales, An introduction with applications, Boston, MA: Birkhäuser Boston Inc. (2001).

Bohner, M., and Peterson, A. (eds.) Advances in dynamic equations on time scales, Boston, MA: Birkhäuser Boston Inc. (2003).

Caputo, M.C. (2009), "Time scales: from nabla calculus to delta calculus and viceversa via duality," Technical report, arXiv:0910.0085

DaCunha, J.J. (2004), "Lyapunov stability and Floquet theory for nonautonomous linear dynamic systems on time scales," Baylor University.

DaCunha, J.J. (2005), "Stability for time varying linear dynamic systems on time scales," $J$. Comput. Appl. Math., 176, 381-410.

Davis, J.M., Gravagne, I.A., Jackson, B.J., and , IIMarks, R.J. (2009), "Controllability, observability, realizability, and stability of dynamic linear systems," Electron. J. Differential Equations, No. 37, 32 pp.

Ferreira, R.A.C., and Torres, D.F.M. (2008), "Higher-order calculus of variations on time scales," in Mathematical control theory and finance Berlin: Springer, pp. 149-159. arXiv:0706.3141

Goodwin, G.C., Graebe, S.F., and Salgado, M.E., Control System Design, Prentice Hall International (2001).

Gürses, M., Guseinov, G.S., and Silindir, B. (2005), "Integrable equations on time scales," J. Math. Phys., 46, 113510, 22 pp. 
Hilger, S. (1990), "Analysis on measure chains - a unified approach to continuous and discrete calculus," Results Math., 18, 18-56.

Jackson, B.J. (2007), "A General Linear Systems Theory on Time Scales: Transforms, Stability, and Control," PhD thesis, Baylor University.

Jackson, B.J. (2008), "Adaptive control in the nabla setting," Neural Parallel Sci. Comput., 16, $253-272$.

Kalman, R.E., Falb, P.L., and Arbib, M.A., Topics in mathematical system theory, New York: McGraw-Hill Book Co. (1969).

Kotta, Ü., Bartosiewicz, Z., Pawłuszewicz, E., and Wyrwas, M. (2009), "Irreducibility, reduction and transfer equivalence of nonlinear input-output equations on homogeneous time scales," Systems Control Lett., 58, 646-651.

Martins, N., and Torres, D.F.M. (2009), "Calculus of variations on time scales with nabla derivatives," Nonlinear Anal., 71, e763-e773. arXiv:0807.2596

Olsder, G.J., and van der Woude, J.W., Mathematical systems theory. 3rd ed., Delft: VSSD. (2005).

Pawłuszewicz, E., and Torres, D.F.M. (2010), "Avoidance Control on Time Scales," J. Optim. Theory Appl., 145, in press, DOI: 10.1007/s10957-010-9694-1 arXiv:0910.3308

Seiffertt, J., Sanyal, S., and Wunsch, D.C. (2008), "Hamilton-Jacobi-Bellman Equations and Approximate Dynamic Programming on Time Scales," IEEE Transactions on Systems, Man, and Cybernetics-Part B: Cybernetics, 38, 918-923.

Wolovich, W.A., and Elliott, H. (1983), "Discrete models for linear multivariable systems," Internat. J. Control, 38, 337-357.

Zabczyk, J., Mathematical control theory, An introduction, Reprint of the 1995 edition, Modern Birkhäuser Classics, Boston, MA: Birkhäuser Boston Inc. (2008). 